\title{
(Co)variance components and genetic parameters for growth rate and Kleiber ratio in fat-tailed Mehraban sheep
}

\author{
Farhad Ghafouri-Kesbi \\ Islamic Azad University, Karaj Branch, Karaj, Iran
}

\begin{abstract}
The aim of the present study was to estimate (co)variance components and genetic parameters for average daily gain from birth to weaning (ADGa), weaning to 6 months (ADGb), weaning to 9 months (ADGc), 6 months to 9 months (ADGd) and corresponding Kleiber ratios (KRa, $\mathrm{KRb}, \mathrm{KRc}$ and KRd) in Mehraban sheep. A derivative-free algorithm combined with a series of six univariate linear animal models was used to estimate phenotypic variance and its direct, maternal and residual components. In addition, bivariate analyses were done to estimate (co)variance components between traits. Estimates of direct heritability $\left(h^{2}\right)$ were $0.10,0.11$, $0.16,0.09,0.13,0.13,0.15$ and 0.08 for ADGa, ADGb, ADGc, ADGd, KRa, KRb, KRc and KRd, respectively and indicate that in Mehraban sheep genes contribute very little to the variance of the growth rate and Kleiber ratio. Estimates of maternal heritability $\left(\mathrm{m}^{2}\right)$ were $0.10,0.08$ and 0.05 for $\mathrm{ADGa}$, KRa and $\mathrm{KRb}$, respectively. Direct additive genetic correlations ranged from -0.32 (KRa-KRd) to 0.99 (ADGb-KRb) and phenotypic correlations ranged from -0.53 (ADGaADGd) to 0.99 (ADGa-KRa). Estimates of direct heritability and genetic correlations show that genetic improvement in efficiency of feed utilization through selection programmes is possible, though it would generate a relatively slow genetic progress.
\end{abstract}

Keywords: sheep, growth, Kleiber ratio, animal model, heritability

\begin{abstract}
Abbreviations: ADG: average daily gain, ADGa: ADG from birth to weaning, ADGb: ADG from weaning to 6 months, ADGc: ADG from weaning to 9 months, ADGd: ADG from 6 months to 9 months, AIC: Akaike information criterion, $h^{2}$ : direct heritability, KR: Kleiber ratio, KRa, KRb, KRc, KRd: corresponding Kleiber ratios for ADGa-ADGc, $m^{2}$ : maternal heritability, $s_{a}^{2}$ : direct additive genetic variance, $s_{c}^{2}$ : maternal permanent environmental variance, $s_{m}^{2}$ : maternal genetic variance, $s_{a, m}$ : direct-maternal additive genetic covariance, $s_{\mathrm{e}}^{2}$ : residual variance
\end{abstract}

Archiv Tierzucht 56 (2013) 55, 564-572

doi: 10.7482/0003-9438-56-055

Corresponding author:

Farhad Ghafouri-Kesbi; email: farhad_ghy@yahoo.com

Islamic Azad University, Karaj Branch, P. O. Box: 31485-313, Karaj, Iran
Received: 7 January 2013

Accepted: 1 March 2013

Online: 26 April 2013

() 2013 by the authors; licensee Leibniz Institute for Farm Animal Biology (FBN), Dummerstorf, Germany. This is an Open Access article distributed under the terms and conditions of the Creative Commons Attribution 3.0 License (http://creativecommons.org/licenses/by/3.0/). 


\section{Introduction}

In 2007, the sheep population in Iran reached 25 million heads (Sefidbakht 2011). This large population is mainly reared for meat production to provide a part of the protein requirements of a population of 75 million people. In recent decades, many breeding programmes targeting the growth performance have been planned and performed in most of the Iranian sheep breeds. Reviewing the results of these selection programmes reveals noticeable genetic improvement (see Ghafouri-Kesbi et al. 2009, Mokhtari \& Rashidi 2010 for example). However, in Iran sheep production is highly dependent on pasture production. The decrease in winter and spring precipitation diminishes the quantity and quality of the pasture, thereby limiting the efficiency of selection programmes. In addition, reduction in pasture area in the last decades have forced the government to withdraw the livestock from forest and range areas and develop intensive systems which in turn significantly increase the cost of sheep production. A strategy to increase the efficiency of meat production in both the traditional and intensive systems is the selection of animals with respect to efficiency of feed utilization. Since individual sheep differ in their ability to efficiently utilize feed, selecting the most efficient animals, those with lower maintenance requirements, results in a significantly lower production cost (Ghafouri-Kesbi et al. 2011). Direct selection for lower maintenance requirements is difficult. However, measures of feed efficiency such as Kleiber ratio (KR; Kleiber 1947) would be used to achieve this goal (Arthur et al. 2001). The KR, defined as growth rate divided by body mass ${ }^{0.75}$ (metabolic weight), has been recommended as an indirect selection parameter for feed conversion. Results show that a part of variation among animals concerning the KR is heritable $\left(h^{2}=0.01-0.15\right)$. In addition, Arthur et al. (2001) found a strong correlation between KR and feed conversion ratio in bulls $(-0.81)$. Furthermore, there is a positive genetic correlation between KR with traits related to growth (Abegaz 2005, Ghafouri-Kesbi et al. 2011). These findings show that improvements in feed efficiency could be made without negatively affecting body weight or growth rate.

Mehraban sheep is one of the important Iranian sheep breeds, numbering approximately 3 million heads and is adapted to harsh and rocky environment in the western parts of the country. Today, a large percentage of the Mehraban sheep population is raised in the Hamadan province. The breed is fat-tailed, large-sized, has carpet-wool with light brown, cream or grey colour and is primarily used for meat production. Due to the large population of Mehraban sheep, there is an increasing interest for genetic improvement of this breed.

In order to make genetic improvement in growth rate and Kleiber ratio, knowledge of genetic parameters such as heritability and genetic correlations among traits are required. This genetic information is used to construct efficient selection indexes to achieve genetic improvement in the traits of interest through selection programmes. Although genetic parameters for growth traits and Kleiber ratio have been estimated for some breeds of sheep (Abegaz 2005, Mohammadi et al. 2010, Jafaroghli et al. 2010, Ghafouri-Kesbi et al. 2011, SavarSofla et al. 2011, Mokhtari et al. 2012), no previous information on the genetic parameters of growth rate and Kleiber ratio in Mehraban sheep is available. Therefore, the aim of this paper was to estimate necessary genetic parameters for growth rate and Kleiber ratio for Iranian Mehraban sheep. 


\section{Material and methods}

\section{Data}

Live weight records of Mehraban lambs were used in this study. The data were obtained from the RAMPLANE database of the Mehraban sheep breeding unit of the Agriculture Organization of the Hamadan province. For genetic improvement of Mehraban sheep and increasing its growth performance, the RAMPLANE project was started in spring 1989 by the Agriculture Ministry of Iran. In this project, artificial insemination (Al), which is in progress, is used to conceive ewes with semen of selected sires. Artificial insemination makes genetic connectedness among flocks which is necessary for accurate genetic evaluation. Most of the lambing takes place in March and April. During the lambing season, the ewes are indoors and carefully managed. At lambing, lambs are weighed and ear-tagged within 12 hours after birth. During the suckling period, lambs are fed with ewes' milk and also allowed to access dry alfalfa at 3 weeks of age. Lambs are weaned for 90 days on average. Animals are maintained under semi-extensive farm conditions and generally allowed to graze on range which is medium in vegetation of such genera as Artemisia, Astragalus, Brumus and Stipa. Range conditions are poor during the late autumn and winter months and therefore, the sheep receive supplemental feed for 6 months of the year. During this period, sheep have ad libitum access to alfalfa hay and some concentrates. Ewes are kept in the flock for a maximum of six parities. However, ewes that did not lamb in any season as well as sick ewes are culled sooner. Selection of young rams is based on body weight and body conformation.

\section{Traits considered}

The increases in weight for the different growth phases, namely birth to weaning, weaning to 6 months, weaning to 9 months and 6 months to 9 months of age were used to calculate ADGa, ADGb, ADGc and ADGd, respectively, as total gain divided by the number of days in the period. These were used to calculate corresponding Kleiber ratios as $\mathrm{KRa}=\mathrm{ADGa} / \mathrm{WW}^{0.75}$, $\mathrm{KRb}=\mathrm{ADGb} / \mathrm{W6}^{0.75}, \mathrm{KRc}=\mathrm{ADGc} / \mathrm{W} 9^{0.75}$ and $\mathrm{KRd}=\mathrm{ADGd} / \mathrm{W} 9^{0.75}$.

\section{Statistical analysis}

To identify fixed effects to be included in the models, least square analyses were conducted using the GLM procedure of SAS 9 (SAS Institute Inc., Cary, NC, USA). This was performed on a model including fixed effects of herd, year of birth, season of birth, age of dam, sex of lambs and type of birth. All these fixed effects were significant $(P<0.05)$ for all traits and were included in the models. A series of six univariate linear animal models was considered that differed in the (co)variance components fitted to assess the importance of maternal effects (Table 2). The models expanded from a simple animal model (Model 1) to a comprehensive maternal effects model (Model 6). The general representation of the most complete animal model (Model 6) used in the analyses was as follows:

$$
y=X \beta+Z_{1} a+Z_{2} c+Z_{3} m+e_{1} \operatorname{Cov}(a, m)=A s_{a, m}
$$


where $y$ is the vector of phenotypic observations for each trait and $\beta$ is the vector of fixed effects to be fitted with associated matrix $X$. The vector $a$ contains the direct additive genetic effects for each individual $\left(a_{i}\right)$ having a mean of zero and a variance of $s_{a}^{2}$. This is estimated from the variance-covariance matrix of additive genetic effects $(G)$ which is equal to $A s_{a^{\prime}}^{2}$ where $A$ is the additive numerator relationship matrix created from the pedigree structure. Similarly, maternal genetic variance $\left(s_{m}^{2}\right)$ and maternal permanent environment variance $\left(s_{c}^{2}\right)$ were estimated by including $m$ and $c$, the vectors of maternal genetic and maternal permanent environment effects, respectively. The variance-covariance matrix of maternal genetic effects is specified as $A s_{m}^{2}$ such that estimating $s_{m}^{2}$ uses the relationship matrix in the same way as the additive genetic effect. $s_{a, m}$ denotes the covariance between direct and maternal genetic effects. In all models, $e$ was fitted as the vector of residual errors (corresponding to temporary environment effects) with variance of $s_{e}^{2}$. Maternal permanent environmental effects and residual errors were assumed to be normally distributed with means of 0 and variance-covariance matrices of $I_{n d} s_{c}^{2}$ and $I_{n} s_{e}^{2}$, where $I_{n d}$ and $I_{n}$ are identity matrices of order equal to the number of dams and number of records, respectively. The direct additive genetic effects, maternal permanent environmental effects and maternal genetic effects were related to individual records with the corresponding incidence matrices $Z_{1}, Z_{2}$ and $Z_{3^{\prime}}$ respectively.

Estimation of (co)variance components was carried out using a derivative-free algorithm using the DFREML-package (Meyer 2001). The Akaike information criterion (AIC) of Akaike (1973) was computed to rank the models. Let $p$ denote the number of random (co)variance parameters to be estimated and $\log L$ the maximum likelihood, then the information criterion is defined as: $A I C=-2 \log L+2 p$. The model yielding the smallest AIC fits the data best.

(Co)variance components between traits were estimated from bivariate analyses. The models applied in bi-variate analyses were those fitted for each of the underlying traits in the univariate analyses.

\section{Results and discussion}

Mean, standard deviation and coefficient of variation of traits studied with a summary of pedigree structure are shown in Table 1. As shown, in the pre-weaning period animals have the maximum growth rate (191.3 g/day). Ghafouri-Kesbi et al. (2011), Savar-Sofla et al. (2011) and Mokhtari et al. (2012) reported similar findings. The same results were observed regarding the Kleiber ratio, with a maximum in the pre-weaning growth phase concomitant with the less coefficient of variance. Maternal care and the genetic potential of lambs are two factors which affect the early growth of lambs. In the pre-weaning period, animals are fed by their mother's milk and are less affected by environmental conditions. It reflects in minimum phenotypic $(C V=19.8)$ which shows less responsiveness to environmental effects. After weaning, lambs lose maternal support and their growth depends on availability of feed and grazing resources. Unfavourable environmental conditions decrease feed resources, thereby limiting the lambs' growth. As a result, differences among lambs' performance regarding growth and efficiency of feed utilization cannot be realized very well during the pre-weaning growth phase because good maternal care can cover a poor genetic potential of lambs and vice versa. 
Table 1

Characteristics of the data structure

\begin{tabular}{lcccccccc}
\hline & ADGa & ADGb & ADGc & ADGd & KRa & KRb & KRc & KRd \\
\hline No. records & 2065 & 1560 & 1060 & 740 & 2065 & 1560 & 1060 & 740 \\
No. of sire & 91 & 88 & 74 & 75 & 91 & 88 & 74 & 75 \\
No. of dam & 651 & 595 & 410 & 416 & 651 & 595 & 410 & 416 \\
Mean, g & 191.3 & 152.5 & 64.1 & 37.8 & 19.61 & 12.21 & 7.81 & 4.31 \\
Standard deviation, g & 38.5 & 40.3 & 22.3 & 16.1 & 2.4 & 1.6 & 2.8 & 1.9 \\
Coefficient of variation, \% & 19.8 & 26.5 & 34.8 & 42.5 & 12.2 & 12.21 & 35.8 & 43.3 \\
\hline
\end{tabular}

Table 2

The random (co)variance components used in the six models

\begin{tabular}{lccccc}
\hline Model number & $\mathrm{s}_{\mathrm{a}}^{2}$ & $\mathrm{~s}_{\mathrm{c}}^{2}$ & $\mathrm{~s}_{\mathrm{m}}^{2}$ & $\mathrm{~s}_{\mathrm{a}, \mathrm{m}}$ & $\mathrm{s}_{\mathrm{e}}^{2}$ \\
\hline 1 & $\checkmark$ & & & & $\checkmark$ \\
2 & $\checkmark$ & $\checkmark$ & & & $\checkmark$ \\
3 & $\checkmark$ & & $\checkmark$ & & $\checkmark$ \\
4 & $\checkmark$ & & $\checkmark$ & $\checkmark$ & $\checkmark$ \\
5 & $\checkmark$ & $\checkmark$ & $\checkmark$ & & $\checkmark$ \\
6 & $\checkmark$ & $\checkmark$ & $\checkmark$ & $\checkmark$ & $\checkmark$ \\
\hline
\end{tabular}

Estimates of variance components and corresponding genetic parameters for traits studied are presented in Table 3 . With respect to growth rate, estimates of direct heritability $\left(h^{2}\right)$ were $0.10,0.11,0.16$ and 0.09 for ADGa, ADGb, ADGc and ADGd, respectively, which are in the range of other reports (El Fadili et al. 2000, Miraei-Ashtiani et al. 2007, Jafaroghli et al. 2010, Ghafouri-Kesbi et al. 2011, Savar-Sofla et al. 2011, Mokhtari et al. 2012). For the pre-weaning growth rate, estimates of direct heritability ranged between 0.03 in Sangsari sheep (MiraeiAshtiani et al. 2007) to 0.46 in Moroccan-Timahdit sheep (El Fadili et al. 2000), though more estimates are lower than 0.2 (Eskandarinasab et al. 2010, Ghafouri-Kesbi et al. 2011, SavarSofla et al. 2011). Literature estimates of direct heritability for post-weaning growth rate has a more broad range from 0.02 in Moghani sheep (Savar-Sofla et al. 2011) to 0.70 in Menz sheep (Gizaw \& Jushi 2004) with higher estimates in crossbred populations (see Gizaw \& Jushi 2004 for example).

For KRa, KRb, KRc and KRd, estimates of $h^{2}$ were $0.13,0.13,0.15$ and 0.08 , respectively, which fall in the range reported for different breeds of sheep (0.01-0.15; Mohammadi et al. 2010, Ghafouri-Kesbi et al. 2011, Savar-Sofla et al. 2011, Mokhtari et al. 2012). For pre-weaning KR, estimates of $h^{2}$ range from 0.04 (Arman sheep, Mokhtari et al. 2012) to 0.15 (Sanjabi sheep, Mohammadi et al. 2010) and for post-weaning KR estimates of $h^{2}$ range between 0.01 (Moghani sheep, Savar-Sofla et al. 2011) and 0.10 (Zandi sheep, Ghafouri-Kesbi et al. 2011). Albeit, estimates of heritability for a trait can differ between sheep breeds and may change slowly over time. Literature estimates of $h^{2}$ reveal that in sheep, the Kleiber ratio is a substantially low heritable trait. In goat, reports are a bit higher than in sheep (0.13-0.35, Van Niekerk et al. 1994, Supakorn \& Pralomkarn 2012). In beef cattle, higher estimates of $h^{2}$ 
for Kleiber ratio have been reported. For example, Köster et al. (1994) reported estimates of $h^{2}$ ranging from 0.22 (KR at 205 days of age) to 0.54 (KR at 365 days of age) in Herford breed. These results show that estimates of heritability are both breed-and species-specific.

According to current results, pre- and post-weaning growth rate and Kleiber ratio in Mehraban sheep are categorized as low heritable traits. Therefore, a low genetic progress would be expected through selection programmes. If a trait is only $10 \%$ heritable, then $90 \%$ of differences between animals would have been of non-genetic origin. For low heritable traits, where $h^{2}$ is below 0.15 , an animal's performance is much less useful for identifying the individuals with the best genes for the trait (Cassel 2009). For this reason, selection for both growth rate and Kleiber ratio in Mehraban sheep should be according to estimates of breeding values (Janssens \& Vandepitte 2004).

Among the traits studied ADGa, KRa and KRb were affected by maternal effects. Estimates of maternal genetic effects (maternal heritability, $\mathrm{m}^{2}$ ) were $0.10,0.08$ and 0.05 for ADGa, KRa and $\mathrm{KRb}$, respectively. These findings are in consonance with other reports (Abegaz 2005, Szwaczkowski et al. 2006, Mohammadi et al. 2010, Ghafouri-Kesbi et al. 2011, Savar-Sofla et al. 2011, Mokhtari et al. 2012). Maternal genetic effects arise from allelic differences between individual mothers at loci influencing offspring phenotype and are a heritable component of phenotypic variance themselves. It can dampen (when direct maternal genetic covariance is negative) or accelerate (when direct maternal genetic covariance is positive) the response to selection (Wolf et al. 1998). As shown in Table 3, for ADGa maternal heritability is equal to direct heritability ( 0.10 vs. 0.10$)$. It shows that genes contributing to maternal performance have an equal influence on the early growth of lambs as genes carried by lambs. If maternal genetic effects are not considered, the true genetic potential of lambs is masked by maternal performance making it difficult to select superior animals. As a result, maternal effects should be included in the model to obtain accurate estimates of genetic parameters and to have accurate estimates of breeding values, in particular where animals are evaluated for traits related to early growth.

Table 3

Variance components and heritability estimates for traits studied

\begin{tabular}{lcccrrrc}
\hline Trait & Model & \multicolumn{1}{c}{$\mathrm{s}_{\mathrm{a}}^{2}$} & $\mathrm{~s}_{\mathrm{m}}^{2}$ & \multicolumn{1}{c}{$\mathrm{s}_{\mathrm{e}}^{2}$} & \multicolumn{1}{c}{$\mathrm{s}_{\mathrm{p}}^{2}$} & $\mathrm{~h}^{2}$ & $\mathrm{~m}^{2}$ \\
\hline ADGa & 3 & 102.51 & 99.63 & 800.44 & 1002.58 & $0.10(0.03)$ & $0.10(0.04)$ \\
ADGb & 1 & 142.65 & & 1124.51 & 1267.16 & $0.11(0.03)$ & - \\
ADGc & 1 & 37.42 & & 196.50 & 233.92 & $0.16(0.04)$ & - \\
ADGd & 1 & 14.39 & & 160.41 & 174.80 & $0.09(0.04)$ & - \\
KRa & 3 & 0.21 & 0.13 & 1.21 & 1.66 & $0.13(0.03)$ & $0.08(0.03)$ \\
KRb & 3 & 0.64 & 0.24 & 4.03 & 4.91 & $0.13(0.02)$ & $0.05(0.03)$ \\
KRc & 1 & 0.09 & & 0.53 & 0.62 & $0.15(0.03)$ & - \\
KRd & 1 & 0.04 & & 0.43 & 0.47 & $0.08(0.03)$ & - \\
\hline
\end{tabular}

Estimates of different correlations between traits are presented in Table 4. Direct additive genetic correlation between traits ranged from -0.32 (KRa-KRd) to 0.98 (ADGa-KRa) and 
phenotypic correlations ranged from -0.53 (ADGa-ADGb) to 0.98 (ADGa-KRa) in agreement with Ghafouri-Kesbi et al. (2011), Savar-Sofla et al. (2011) and Mokhtari et al. (2012). Genetic and phenotypic correlations decrease as the age distance between measures increases. In particular, the genetic correlation between adjacent measures approaches unity. Positive correlation means that selection for growth rate not only improves the growth rate of animals but also the efficiency of feed utilization. The genetic correlation of traits is independent of their heritability, i.e. two traits can have a very high genetic correlation even when the heritability of each is low. However, when the difference between heritability of two traits is

Table 4

Correlations between traits studied yielded from bivariate analyses

\begin{tabular}{|c|c|c|c|c|}
\hline Trait 1 & Trait 2 & $r_{a}$ & $r_{m}$ & $r_{p}$ \\
\hline ADGa & $\mathrm{ADGb}$ & $0.94(0.11)$ & - & 0.77 \\
\hline ADGa & $A D G C$ & $0.24(0.09)$ & - & 0.33 \\
\hline ADGa & ADGd & $-0.18(0.04)$ & - & -0.53 \\
\hline $\mathrm{ADGa}$ & KRa & $0.91(0.11)$ & $0.88(0.08)$ & 0.98 \\
\hline ADGa & $\mathrm{KRb}$ & $0.54(0.13)$ & $0.63(0.09)$ & 0.48 \\
\hline ADGa & KRc & $0.23(0.10)$ & - & -0.13 \\
\hline ADGa & KRd & $-0.31(0.03)$ & - & -0.37 \\
\hline ADGb & $A D G C$ & $0.64(0.07)$ & - & 0.40 \\
\hline$A D G b$ & ADGd & $0.39(0.11)$ & - & 0.53 \\
\hline$A D G b$ & KRa & $0.84(0.14)$ & - & 0.89 \\
\hline ADGb & $\mathrm{KRb}$ & $0.99(0.05)$ & - & 0.93 \\
\hline ADGb & KRc & $0.66(0.12)$ & - & 0.65 \\
\hline ADGb & KRd & $0.32(0.05)$ & - & 0.34 \\
\hline ADGc & ADGd & $0.29(0.08)$ & - & 0.18 \\
\hline ADGC & KRa & $0.44(0.13)$ & - & 0.31 \\
\hline ADGc & $\mathrm{KRb}$ & $0.43(0.10)$ & - & 0.17 \\
\hline ADGC & KRc & $0.92(0.02)$ & - & 0.77 \\
\hline ADGC & KRd & $0.76(0.09)$ & - & 0.56 \\
\hline ADGd & KRa & $-0.08(0.12)$ & - & -0.43 \\
\hline ADGd & $\mathrm{KRb}$ & $0.35(0.07)$ & - & 0.41 \\
\hline ADGd & KRc & $0.43(0.13)$ & - & 0.22 \\
\hline ADGd & KRd & $0.86(0.22)$ & - & 0.64 \\
\hline KRa & $\mathrm{KRb}$ & $0.96(0.15)$ & - & 0.77 \\
\hline KRa & KRc & $0.14(0.08)$ & - & 0.13 \\
\hline KRa & KRd & $-0.32(0.13)$ & - & -0.29 \\
\hline $\mathrm{KRb}$ & KRc & $0.45(0.17)$ & - & 0.62 \\
\hline $\mathrm{KRb}$ & KRd & $0.54(0.10)$ & - & 0.39 \\
\hline KRc & KRd & $0.09(0.09)$ & - & 0.40 \\
\hline
\end{tabular}

$r_{a}$ : direct additive genetic correlation, $r_{m}$ : maternal genetic correlation, $r_{p}$ : phenotypic correlation 
high, the genetic correlation will be overestimated and should be treated with caution. The genetic and phenotypic correlations between ADGa with KRa and KRb and between ADGb with $\mathrm{KRa}$ and $\mathrm{KRb}$ are highly positive, indicating that lambs with a higher growth rate are supposed to be more efficient users of feed and by selecting them, the biological efficiency of the flock will be improved.

In conclusion, traits studied experienced low additive genetic variation. In spite of this, they can be used in a selection index to select superior animals because in addition to the heritability, genetic correlation between the interested trait with other traits and economic weight of the trait are important to allow a trait to be included in the selection index. The high economic weight of the trait can compensate its low heritability. Finally, since traits have a low heritability, selection of animals should be based on their breeding values.

\section{Acknowledgements}

Valuable comments of two anonymous reviewers on an earlier draft of this manuscript are gratefully acknowledged.

\section{References}

Abegaz S, van Wyk, JB, Olivier JJ (2005) Model comparisons and genetic and environmental parameter estimates of growth and the Kleiber ratio in Horro sheep. S Afr J Anim Sci 35, 30-40

Akaike H (1973) Information theory and an extension of the maximum likelihood principle. In: Petrov BN, Csáki F (eds.) Proc 2nd Int Symp Info Theory, Tsahkadsor, Armenia, USSR September 2-8, 1971. Akademiai Kiado, Budapest, Hungary, 267-281

Arthur PF, Renand G, Krauss D (2001) Genetic and phenotypic relationships among different measures of growth and feed efficiency in young Charolais bulls. Livest Prod Sci 68, 131-139

Cassel B (2009) Using Heritability for Genetic Improvement. Virginia Cooperative Exteension, Publication 404-084, College of Agriculture and Life Sciences. Virginia Polytechnic Institute and State University, USA http://pubs.ext.vt.edu/404/404-084/404-084_pdf.pdf [last accessed 20.11.2013]

El Fadili M, Michaux C, Detilleux J, Leroy PL (2000) Genetic parameters for growth traits of the Moroccan Timahdit breed of sheep. Small Rumin Res 37, 203-208

Eskandarinasab M, Ghafouri-Kesbi F, Abbasi MA (2010) Different models for evaluation of growth traits and Kleiber ratio in an experimental flock of Iranian fat-tailed Afshari sheep. J Anim Breed Genet 127, 26-33

Gizaw S, Joshi BK (2004) Estimates of genetic parameters of growth traits in Menz and Awassi $\times$ Menz crossbred sheep in Ethiopia. Indian J Anim Sci 74, 864-867

Ghafouri-Kesbi F, Eskandarinasab MP, Hassanabadi A (2009) Short-term selection for yearling weight in a small experimental Iranian Afshari sheep flock. Can J Anim Sci 89, 301-307

Ghafouri-Kesbi F, Abbasi MA, Afraz F, Babaei M, Baneh H, Abdollahi Arpanahi R (2011) Genetic analysis of growth rate and Kleiber ratio in Zandi sheep. Trop Anim Health Prod 43, 1153-1159

Jafaroghli M, Rashidi A, Mokhtari MS, Shadparvar AA (2010) (Co)Variance components and genetic parameter estimates for growth traits in Moghani sheep. Small Rumin Res 91, 170-177

Janssens S, Vandepitte W (2004) Genetic parameters for body measurements and linear type traits in Belgian Blue du Maine, Suffolk and Texel sheep. Small Rumin Res 54, 13-24

Kleiber M (1947) Body size and metabolic rate. Physiol Rev 27, 511-541

Köster E, van der Westhuizen J, Erasmus GJ (1994) Heritability estimates for different Kleiber ratios obtained from growth performance data in a Hereford herd. S Afr J Anim Sci 24, 71-72 
Meyer K (2001) Programs to estimate variance components for individual animal models by restricted maximum likelihood (REML). Ver. 3.1.0. Users notes. Institute of Animal Science. Animal Genetics and Breeding Unit, University of New England, Armidale, NSW, Australia

Miraei-Ashtiani SR, Seyedalian SAR, Moradi Shahrbabak M (2007) Variance components and heritabilities for body weight traits in Sangsari sheep, using univariate and multivariate animal models. Small Rumin Res 73, 109-114

Mohammadi Y, Rashidi A, Mokhtari MS, Esmailizadeh AK (2010) Quantitative genetic analysis of growth traits and Kleiber ratios in Sanjabi sheep. Small Rumin Res 93, 88-93

Mokhtari MS, Rashidi A (2010) Genetic trends estimation for body weights of Kermani sheep at different ages using multivariate animal models. Small Rumin Res 88, 23-26

Mokhtari MS, Moradi Shahrebabak M, Moradi Shahrebabk H, Sadeghi M (2012) Estimation of (co)variance components and genetic parameters for growth traits in Arman sheep. J Livest Sci Tech, 38-47

Savar-Sofla S, Nejati-Javaremi A, Abbasi MA, Vaez-Torshizi R, Chamani M (2011) Investigation on Direct and Maternal Effects on Growth Traits and the Kleiber Ratio in Moghani Sheep. World Appl Sci J 14, 1313-1319

Szwaczkowski T, Wójtowski J, Stanisławska E, Gut A (2006) Estimates of maternal genetic and permanent environmental effects in sheep. Arch Tierz 49, 186-192

Sefidbakht N (2011) Future aspect of sustainable animal production in Iran, focusing on the sheep and goat. The First Seminar on Animal Production in Tropical Environment. Krman University, Iran, 1-9

Supakorn C, Pralomkarn W (2012) Genetic parameter estimates for weaning weight and Kleiber ratio in goats. Songklanakarin J Sci Technol 34, 165-172

Van Niekerk MM, Schoeman SJ, Botha ME, Casey N (1996) Heritability estimates for pre-weaning growth traits in the Adelaide [South Africa] Boer goat flock. S Afr J Anim Sci 26, 6-10

Wolf JB, Brodie ED, Cheverud JM, Moore AJ, Wade MJ (1998) Evolutionary consequences of indirect genetic effects. Trends Ecol Evol 13, 64-69 